\title{
Ballistic and diffusive corrections to front propagation in the presence of multiplicative noise
}

\author{
J. Armero, ${ }^{1}$ J. Casademunt,${ }^{1}$ L. Ramírez-Piscina, ${ }^{2}$ and J. M. Sancho ${ }^{1,3}$ \\ ${ }^{1}$ Departament d'Estructura i Constituents de la Matèria, Universitat de Barcelona, Avenida Diagonal 647, \\ E-080028 Barcelona, Spain \\ ${ }^{2}$ Departament de Física Aplicada, Universitat Politècnica de Catalunya, Avenida Dr. Gregorio Marañón 50, \\ E-08028 Barcelona, Spain \\ ${ }^{3}$ Institute for Nonlinear Science, University of California at San Diego, 9500 Gilman Drive, Department 0402, \\ La Jolla, California 92093-0402
}

(Received 29 May 1998)

\begin{abstract}
We study the dynamics of reaction-diffusion fronts under the influence of multiplicative noise. An approximate theoretical scheme is introduced to compute the velocity of the front and its diffusive wandering due to the presence of noise. The theoretical approach is based on a multiple scale analysis rather than on a small noise expansion and is confirmed with numerical simulations for a wide range of the noise intensity. We report on the possibility of noise sustained solutions with a continuum of possible velocities, in situations where only a single velocity is allowed without noise. [S1063-651X(98)03611-3]
\end{abstract}

PACS number(s): 05.40.+j, 47.54.+r, 03.40.Kf, 47.20.Ky

\section{INTRODUCTION}

The role of fluctuations in spatially extended systems is a subject of current interest in a great variety of out of equilibrium systems [1]. In particular, a class of problems which has received considerable attention is that of fronts propagating between states of different stability. This generic problem is relevant to a large variety of systems in nonlinear physics, chemistry, and biology. The effects of noise on front propagation have been studied from different points of view [2-6]. In this paper we address the problem of fronts propagating in media where fluctuations have been externally imposed through randomness of some external control parameter. We make use of a Langevin formalism, i.e., the model is formulated in terms of nonlinear partial differential equations which contain noise-source terms which in general are multiplicative $[2,3,7]$.

It is commonly believed that the effects of external fluctuations in front propagation, provided they enter multiplicatively in the equations, are twofold: first, they induce a shift in the mean front velocity, and second, they produce a random wandering of the front position with respect to its mean position. Concerning the mean front velocity, the problem reduces to an analogous deterministic problem with renormalized coefficients [6]. This is a very useful result because the mean front velocity can then be analyzed within the usual scenario of deterministic front propagation [8]. This implies that the boundaries in parameter space separating the different qualitative behaviors are preserved but shifted by noise. As a consequence, noise not only produces a quantitative change in the front velocity but can change the qualitative behavior of the front inducing a transition to a different region of the parameter space.

Here we extend the formalism of Ref. [6] to permit the study of the wandering of the front position. This wandering is assumed to be diffusive. Our approach provides explicit predictions of the associated diffusion coefficient which go beyond previous results that were first-order expansions in the noise intensity $[7,2,3]$. Our approach is not perturbative in the noise intensity, but is closer to a (stochastic) multiple scale analysis. We formally assume a separation of scales which introduces a small parameter, and propose a certain scaling of the different contributions to the front dynamics. The procedure implicitly assumes a partial summation of the expansion on the noise intensity, and is therefore not truly systematic. However, we show with numerical simulations that the obtained results are remarkably good even for relatively large noise intensities, and that the physical picture behind the analysis is correct within a broad range of parameters. A remarkable example of this is that noise can sustain solutions with a continuum of possible front velocities (linear scenario of front selection) in cases which have a single possible velocity in the absence of noise (nonlinear scenario). The theoretical framework is also suitable to assess the validity of the diffusive assumption of the front wandering and predicts self-consistently its failure in some parameter regimes.

We are interested in the situation in which a front is described by a field $\phi(x, t)$ with a globally stable state (e.g., $\phi=\phi_{\text {st }}$ ) invading another (unstable or metastable) state (e.g., $\phi=0)$. When external fluctuations are imposed, simulations show [6] that the kinklike structure of the front is not destroyed even for moderately large noise intensities. Consequently, these noisy fronts have a rather well defined position and average shape. The location of a front invading a $\phi=0$ region can be defined by the integral

$$
z(t)=\frac{1}{\phi_{s t}} \int_{x_{0}}^{\infty} d x \phi(x, t)=\langle z(t)\rangle-\Delta(t),
$$

where $\phi_{s t}$ is the steady state left behind the front and $\langle z(t)\rangle$ is the ensemble average of the front position. $\Delta(t)$ is the instantaneous departure from the average position, and is thus the quantity which models the stochastic wandering of the front. The physical picture behind the present analysis is that for time and length scales in which the front structure is not resolved, the quantity $z(t)$ can be modeled in a very good approximation by a simple stochastic process of the form 


$$
\dot{z}(t)=\bar{v}(\varepsilon)+D^{1 / 2}(\varepsilon) \zeta(t),
$$

where $\zeta(t)$ is a Gaussian white noise with zero mean and correlation $\left\langle\zeta(t) \zeta\left(t^{\prime}\right)\right\rangle=2 \delta\left(t-t^{\prime}\right)$, and where $\varepsilon$ is a measure of the noise intensity in the original equation for the front variable $\phi$. The velocity $\bar{v}(\varepsilon)$ for a given $\varepsilon$ is a constant. With these definitions the quantity $\Delta(t)$ is assimilated to a Wiener process with

$$
\left\langle\Delta^{2}(t)\right\rangle=2 D(\varepsilon) t
$$

The mean velocity $\bar{v}(\varepsilon)$ was the object of study of Ref. [6]. Here we are concerned with the computation of the diffusion of coefficient $D(\varepsilon)$. The present approach will also allow for a self-consistent test of the validity of the above simple physical picture. We will also test the theoretical predictions through numerical simulations. These will be carried out on an ensemble of front realizations where the instantaneous front position is measured as a function of time according to Eq. (1). The coefficient $D(\varepsilon)$ is then evaluated through a linear fit of $\left\langle\Delta^{2}(t)\right\rangle$ vs time, after a short transient.

The outline of this paper is as follows. In Sec. II we develop the theorerical approach. In Sec. III we present the model and the explicit results. Section IV deals with a particular front solution sustained by the noise, and finally in Sec. V some conclusions and comments are made.

\section{THEORETICAL APPROACH}

Let us consider a nonlinear reaction-diffusion equation for the field $\phi(x)$, and suppose that the reactive term $f(\phi)$ depends on an external control parameter $a$. We assume that this parameter fluctuates locally around its mean value according to $a \rightarrow a(x, t)=a+\varepsilon^{1 / 2} \eta(x, t)$, where $\varepsilon$ is a measure of the strengh of the noise $\eta(x, t)$. Considering small fluctuations, we can expand the reaction term up to first order in the noise. In this way the noise appears multiplying a function of the field, and the resulting equation has the general form

$$
\frac{\partial \phi}{\partial t}=\frac{\partial^{2} \phi}{\partial x^{2}}+f(\phi)+\varepsilon^{1 / 2} g(\phi) \eta(x, t) .
$$

We also assume that the noise $\eta(x, t)$ is Gaussian of zero mean and correlation given by

$$
\left\langle\eta(x, t) \eta\left(x^{\prime}, t^{\prime}\right)\right\rangle=2 C\left(\frac{\left|x-x^{\prime}\right|}{\lambda}\right) \delta\left(t-t^{\prime}\right) .
$$

The parameter $\lambda$ is the characteristic length of the spatial correlation of the noise. The function $C(|x| / \lambda)$ is normalized in such a way that if $\lambda$ is taken as finite but much smaller than any other spatial scale of the system, in particular much smaller than the front width, then Eq. (5) can be approximated by the noise correlation of the Gaussian white spectrum

$$
\left\langle\eta(x, t) \eta\left(x^{\prime}, t^{\prime}\right)\right\rangle=2 \delta\left(x-x^{\prime}\right) \delta\left(t-t^{\prime}\right) .
$$

Our aim is to construct an approximate scheme which formally separates the two main effects of noise, namely that on the ballistic displacement of the front and that on its dif- fusive wandering. The subtlety of the problem lies in the fact that noise modifies simultaneously two aspects which are different in nature (ballistic and diffusive propagation), and which cannot be naively associated to the usual separation between deterministic and stochastic forces. The key idea of our approach is that the separation of these effects is related to an actual separation of time scales on the shape fluctuations of the front. On the one hand there are fast fluctuations which generate an average front shape at relatively small time scales. A temporal coarse graining would thus eliminate these fast fluctuations providing an averaged front shape which is distinct from the deterministic one, and therefore having a different velocity. On the other hand, the residual slow fluctuations of the front shape will then be responsible for the diffusive wandering.

A crucial feature of multiplicative noise is that the mean value of the noise term in the Langevin equation is nonzero, even though the noise itself has zero mean. This produces the so-called spurious drift. The mean value of the noise term can be worked out using Novikov's theorem for Gaussian noises [9], and gives

$$
\varepsilon^{1 / 2}\langle g(\phi) \eta(x, t)\rangle=\varepsilon C(0)\left\langle g^{\prime}(\phi) g(\phi)\right\rangle .
$$

According to this result Eq. (4) can be rewritten in a more useful form,

$$
\frac{\partial \phi}{\partial t}=\frac{\partial^{2} \phi}{\partial x^{2}}+h(\phi)+\varepsilon^{1 / 2} R(\phi, x, t),
$$

where

$$
h(\phi)=f(\phi)+\varepsilon C(0) g^{\prime}(\phi) g(\phi)
$$

and

$$
R(\phi, x, t)=g(\phi) \eta(x, t)-\varepsilon^{1 / 2} C(0) g^{\prime}(\phi) g(\phi) .
$$

Taking into account these definitions, $R$ is a stochastic term with zero mean value and correlation

$$
\begin{aligned}
& \left\langle R(\phi, x, t) R\left(\phi, x^{\prime}, t^{\prime}\right)\right\rangle \\
& \quad=\left\langle\phi(x, t) \eta(x, t) \phi\left(x^{\prime}, t^{\prime}\right) \eta\left(x^{\prime}, t^{\prime}\right)\right\rangle+O\left(\varepsilon^{1 / 2}\right) .
\end{aligned}
$$

With this rearrangement we already separate a systematic contribution from the noise term and a residual stochastic one. This separation is useful because of the white character of the noise which gives a very simple form of the average of the noise term, with no explicit time dependence. This allows for the definition (9) where the average of the extra term has been eliminated. The resulting equation is thus of the same type as the original one with a noise term which is still multiplicative but with zero mean.

We now assume that the diffusive wandering of the front is much slower than the intrinsic diffusion of the field $\phi$ as defined by the reaction-diffusion equation (8). This means that the formal parameter of the expansion will be the ratio of $D$, that is, the actual quantity we want to determine, to the diffusion coefficient of Eq. (8), which in our case is taken to be 1 . The slow time scale will thus be $D t$. 
We assume that $\phi(x, t)$ has a frontlike structure with a well-defined shape centered in the instantaneous position $z(t)$. It is then convenient to employ the reference frame which moves with the front $\xi=x-z(t)$, in which the front fluctuates around its mean shape. In the framework of our hypothesis of multiple scales we can distinguish the rapid shape fluctuations associated to the temporal scale $t$, which are responsible for the systematic change of both the mean front shape and the mean front velocity, and the slow fluctuations of $\phi(\xi, t)$ that are coupled to the wandering of the front position and that depend on the slow scale $D t$. The way in which we explicitly separate both effects of the noise is by introducing the mean front shape $\phi_{0}(\xi)=\langle\phi(\xi, t)\rangle$ and expand $\phi(\xi, t)$ as

$$
\phi(\xi, t)=\phi_{0}(\xi)+\phi_{1}(\xi, D t),
$$

where $\phi_{1}$ is assumed to be of order $D^{1 / 2}$ and has zero mean. Therefore, we retain only the mean contribution of the rapid fluctuations, and the leading order in $D$ of the slow fluctuations. It is worth remarking that this scheme is not equivalent to a standard small-noise expansion since, for instance, to zeroth order, the front shape $\phi_{0}(\xi)$ contains effects of noise from all orders in the noise intensity.

By writing Eq. (8) in the reference frame which moves with the front and by taking the average, we obtain to leading order in $D$

$$
\frac{d^{2} \phi_{0}}{d \xi^{2}}+\bar{v} \frac{d \phi_{0}}{d \xi}+h\left(\phi_{0}\right)=0
$$

which is the equation for a deterministic front $\phi_{0}(\xi)$ propagating at a constant velocity $\bar{v}=\langle\dot{z}(t)\rangle$. Then, the mean profile and front speed depend on both the external coupling $g(\phi)$ and the effective intensity of the noise $\varepsilon C(0)$ through the effective reaction term, $h\left(\phi_{0}\right)$.

In some cases, Eq. (13) yields an equation similar to the deterministic version of the original model Eq. (4) in the reference frame moving with the front but with renormalized or effective parameters. In other cases, depending on the nature of the external coupling $g(\phi)$, Eq. (13) introduces new nonlinearities. In any case, at this point one can in principle use the standard recipes to calculate the selected mean profile and velocity of the front by using the known results for deterministic equations of this type $[8,6]$.

To proceed to next order, we compare Eq. (13) to Eq. (8) and we conclude that the lowest order of the stochastic term $\varepsilon^{1 / 2} R(\phi, \xi, t)$ is $D^{1 / 2}$. Therefore, the order $D^{1 / 2}$ of Eq. (8) is given by

$$
\mathcal{L}\left(\phi_{1}\right)=\varepsilon^{1 / 2} R\left(\phi_{0}, \xi, t\right)-\dot{\Delta}(t) \frac{d \phi_{0}}{d \xi},
$$

where the linear operator $\mathcal{L}$ is defined as

$$
\mathcal{L}=\left[\frac{d^{2}}{d \xi^{2}}+\bar{v} \frac{d}{d \xi}+h^{\prime}\left(\phi_{0}\right)\right]
$$

Differentiating Eq. (13) with respect to $\xi$, we have that $\mathcal{L}\left(d \phi_{0} / d \xi\right)=0$, showing that due to the translational invariance of Eq. (13), $d \phi_{0} / d \xi$ is a (right) eigenfunction with a zero (right) eigenvalue of the (non-self-adjoint) linear operator $\mathcal{L}$. Because $\mathcal{L}$ has vanishing eigenvalues, we must impose a solvability condition requiring than the right-hand side of Eq. (14) should not drive any eigenfunction with zero eigenvalue. Thus, the solvability condition for the existence of a nontrivial solution to Eq. (14) is that the inhomogenous part should be orthogonal to the null space of the adjoint operator $\mathcal{L}^{\dagger}$

$$
\mathcal{L}^{\dagger}=\frac{d^{2}}{d \xi^{2}}-\bar{v} \frac{d}{d \xi}+h^{\prime}\left(\phi_{0}\right)
$$

It can be shown by direct substitution that

$$
\left(\frac{d \phi_{0}}{d \xi}\right)^{\dagger}=e^{\bar{v} \xi} \frac{d \phi_{0}}{d \xi}
$$

is an eigenfunction of $\mathcal{L}^{\dagger}$ of zero eigenvalue. So, the solvability condition for Eq. (14) takes the form

$$
\int_{-\infty}^{\infty} d \xi e^{\bar{v} \xi} \frac{d \phi_{0}}{d \xi}\left(\dot{\Delta}(t) \frac{d \phi_{0}}{d \xi}-\varepsilon^{1 / 2} R\left(\phi_{0}, \xi, t\right)\right)=0 .
$$

Thus, $\dot{\Delta}(t)$ verifies the stochastic differential equation

$$
\dot{\Delta}(t)=\frac{\int_{-\infty}^{\infty} d \xi e^{\bar{v} \xi}\left(d \phi_{0} / d \xi\right) \varepsilon^{1 / 2} R\left(\phi_{0}, \xi, t\right)}{\int_{-\infty}^{\infty} d \xi e^{\bar{v} \xi}\left(d \phi_{0} / d \xi\right)^{2}}
$$

and the diffusion coefficient $D(\varepsilon)$,

$$
\left\langle\Delta^{2}(t)\right\rangle=2 D(\varepsilon) t=\int_{0}^{t} d t^{\prime} \int_{0}^{t} d t^{\prime \prime}\left\langle\dot{\Delta}\left(t^{\prime}\right) \dot{\Delta}\left(t^{\prime \prime}\right)\right\rangle,
$$

is obtained from Eqs. (6), (10), (11), and (19),

$$
D(\varepsilon)=\varepsilon \frac{\int_{-\infty}^{\infty} d \xi e^{2 \bar{v} \xi}\left(d \phi_{0} / d \xi\right)^{2} g^{2}\left(\phi_{0}\right)}{\left[\int_{-\infty}^{\infty} d \xi e^{\bar{v} \xi}\left(d \phi_{0} / d \xi\right)^{2}\right]^{2}},
$$

where higher-order contributions in $\varepsilon$ coming from the correlation (11) have been discarded. The higher-order terms in $\varepsilon$ which are kept in $D(\varepsilon)$ are only those contained in the lowest order in $D(\varepsilon)$, that is, those contained in $\bar{v}$ and $\phi_{0}$.

Equation (21) is the main result of this section and of this paper. It is worth remarking that, in general, the diffusion coefficient $D(\varepsilon)$ is not a simple linear function of the noise intensity $\varepsilon$ due to the nontrivial dependence of the mean propagation speed $\bar{v}$ and of the mean front profile $\phi_{0}$ on the effective noise intensity $\varepsilon C(0)$. In fact, $D(\varepsilon)$ can exhibit a nonmonotonic, bounded, behavior as we shall see in the next section.

\section{EXPLICIT RESULTS FOR A PROTOTYPE MODEL}

The preceding results apply to situations in which fluctuations affect a front propagating between a globally stable state and an unstable or metastable state. We want to have a control parameter to change the relative stability of these two states and in this way to study the role of fluctuations in the different regimes of the deterministic front propagation sce- 
nario studied in the literature [10]. Without loss of generality we take $\phi_{\mathrm{st}}=1$ as the stable state and $\phi=0$ as the unstable or metastable one. A simple expression for the reaction term fulfilling these requirements is a cubic polynomial with two roots at 0 and 1 and a third root, $a$, acting as a control parameter. Explicitly the reaction term reads

$$
f(\phi)=\phi(1-\phi)(\phi+a) .
$$

In this case [we restrict $a$ to the interval $\left.\left(-\frac{1}{2}, 1\right)\right] a$ determines the stability of the invaded $\phi=0$ state with respect to the globally stable state $\phi_{\mathrm{st}}=1$ and, consequently, it also fixes the selection criteria for the asymptotic front speed (linear or nonlinear marginal stability criterion) [8].

Fluctuations both additive and multiplicative can be considered in this problem. It is known that additive noise generates bulk domains of the stable phase ahead of the front and, consequently, a competition process arises between front propagation (short times) and domain growth (long times) [11]. According to Ref. [11], the additive noise does not modify the deterministic scenario of dynamical front selection. As long as the front exists as a transient state, its mean propagation velocity will be determined by the deterministic part of the model equation. Therefore, we will not study here this particular case.

If we assume fluctuations of $a$ as considered in the preceding section, we have, without approximation, external fluctuations of multiplicative type:

$$
\frac{\partial \phi}{\partial t}=\frac{\partial^{2} \phi}{\partial x^{2}}+\phi(1-\phi)(\phi+a)+\varepsilon^{1 / 2} \phi(1-\phi) \eta(x, t) .
$$

It is worth remarking here that the way in which control parameter fluctuations appear is such that it preserves the stationary states $\phi=0$ and $\phi=1$. In this way, the noise term is most important at regions close to the front, but vanishes at the asymptotic states. This permits us to inhibit any domain-growth dynamics that could compete with the front propagation process, in which we are interested here, and therefore the study will not be limited to short times. The new front profile $\phi$ and mean front speed $\bar{v}$ correspond then to the solution of Eq. (13), which for this model reads

$$
\frac{d^{2} \phi_{0}}{d \xi^{2}}+\bar{v} \frac{d \phi_{0}}{d \xi}+\phi_{0}\left(1-\phi_{0}\right)\left(a^{\prime}+c^{\prime} \phi_{0}\right)=0
$$

with the effective parameters

$$
a^{\prime}=a+\varepsilon C(0), \quad c^{\prime}=1-2 \varepsilon C(0) .
$$

Equations (23) and (24) are supplemented with the boundary conditions

$$
\lim _{\xi \rightarrow-\infty} \phi(\xi)=1, \quad \lim _{\xi \rightarrow \infty} \phi(\xi)=0
$$

because the multiplicative noise does not modify the asymptotic steady states of the front.

In this model, the external fluctuations increase the effective value of the control parameter $a$ and simultaneously reduce the coefficient of the highest (cubic) nonlinearity $c$.
That is, the multiplicative noise increases the relative weight of the nonlinearities of the deterministic model. So one expects that the linear-marginal-stability criterion enlarges its range of applicability as the noise intensity increases. As a consequence, we also expect an increase of the propagation velocity.

We apply the ansatz of reduction of order of van Saarloos, from which the mean nonlinear front velocity is given by $[8]$

$$
\bar{v}_{\mathrm{nl}}=\frac{2 a^{\prime}+c^{\prime}}{\sqrt{2 c^{\prime}}}=\frac{2 a+1}{\sqrt{2[1-2 \varepsilon C(0)]}} .
$$

The asymptotic spatial behavior of the corresponding nonlinear solution is given by the single exponential decay $e^{-k_{\mathrm{nl}} \xi}$, with

$$
k_{\mathrm{nl}}=\sqrt{\frac{c^{\prime}}{2}}=\sqrt{\frac{1}{2}-\varepsilon C(0)} .
$$

However, for the control parameter range $a^{\prime}>0$, the application of the linear-marginal-stability criterion for systems evolving from initial step profiles (or with a sufficiently fast decay) shows that the mean linear propagation velocity is

$$
\bar{v}_{l}=2 \sqrt{a^{\prime}}=2 \sqrt{a+\varepsilon C(0)}
$$

with a steady front solution characterized by the asymptotic spatial decay

$$
k_{l}=\sqrt{a^{\prime}}=\sqrt{a+\varepsilon C(0)} .
$$

The transition between the linear and nonlinear regime is given by those values of $a$ satisfying

$$
a=\frac{1}{2}-2 \varepsilon C(0) \text {. }
$$

Moreover, initial slow-decaying profiles with $k_{i}<k_{l}$ in the linear regime and with $k_{i}<k_{l}^{2} / k_{\mathrm{nl}}$ in the nonlinear regime propagate maintaining the initial decay and with an asymptotic velocity

$$
\bar{v}\left(k_{i}\right)=\frac{k_{i}^{2}+a+\varepsilon C(0)}{k_{i}} .
$$

Numerical simulations of the stochastic model of Eq. (23) were done with a standard algorithm for multiplicative noise [12] in a regular one-dimensional lattice with spatial mesh size $\Delta x=0.5$ and time step $\Delta t=10^{-2}$. The effective noise intensity is characterized by its value on the one-dimensional grid,

$$
\varepsilon C(0)=\frac{\varepsilon}{\Delta x} .
$$

This dependence of the effective noise intensity on the mesh size $\Delta x$ used in the numerical simulations is usual in multiplicative noise problems. The fact that noise values are taken as independent for different discretization cells in the numerical algorithm is equivalent to a finite correlation length 


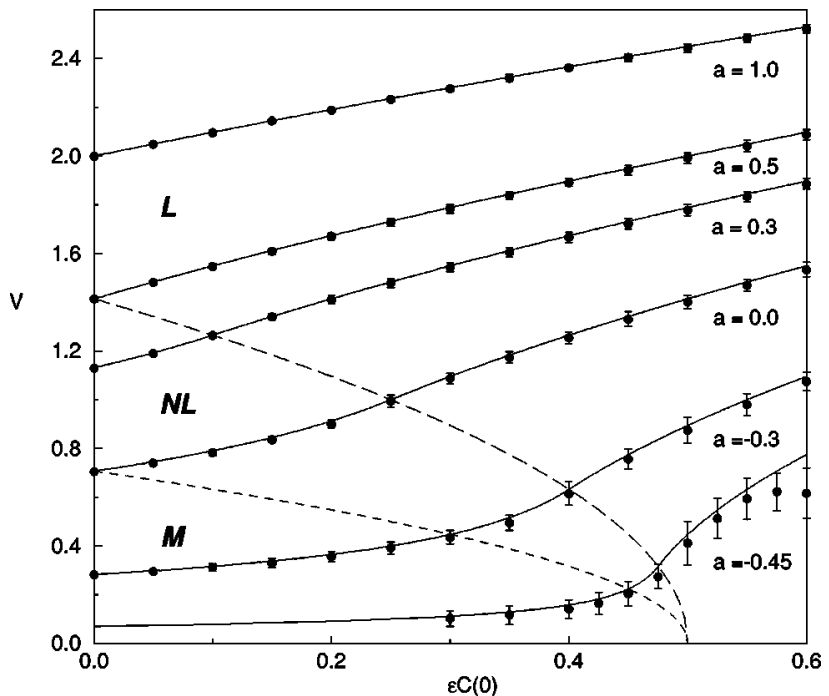

FIG. 1. Mean front velocities as a function of the noise intensity $\varepsilon C(0)$ for several values of the control parameter $a$ of the stochastic model (20). Continuous lines display our analytical predictions (25) and (26). Dashed lines divide the different front regimes: metastable $(M)$, nonlinear $(\mathrm{NL})$, and linear $(L)$. Points and their error bars correspond to numerical simulation.

of the noise of order $\Delta x$. As long as this microscopic cutoff is much smaller than the front width, the noise can be assumed to be white. We will see that, while results for the front velocity depend only on the effective noise intensity as defined by the ratio Eq. (33), the prediction of the front diffusion coefficient $D(\varepsilon)$ depends separately on $\varepsilon$ and $\varepsilon C(0)$, implying that the diffusive dynamics of the front is reminiscent of the existence of such a spatial cutoff.

In Fig. 1 we plot the mean propagation velocity obtained for different values of the control parameter $a$ and the effective noise intensity $\varepsilon C(0)$. The fronts start propagating from initial steplike profiles. The instantaneous front speed is determined by averaging over a time window of size $\tau=200$. Continuous solid lines in this figure display the analytical predictions of Eqs. (27) and (29) showing an extremely good agreement with the numerical results.

In Fig. 2 we compare the analytical prediction of Eq. (21) obtained numerically with simulation results. The agreement between numerical results and the analytical prediction is remarkably good even for large noise intensities. The most surprising feature of our result Eq. (21) is that the dependence of $D(\varepsilon)$ on the effective noise intensity is nonmonotonic, with a maximum value at a finite noise intensity. This is shown explicitly in Fig. 2 for the particular model Eq. (23) with $a=0.1$. The maximum of $D(\varepsilon)$ occurs near $\varepsilon C(0)$ $=0.125$. For a larger value, $\varepsilon C(0)=0.20$, the front spreading is slightly greater during a short initial transient whose duration is associated with the choice of the initial condition, a steplike profile in our case. However, at late time, the spreading is unexpectedly lower than the preceding case, as is seen in Fig. 3.

The nonmonotonic dependence of $D(\varepsilon)$ is the result of the interplay between the two effects of noise, namely modification of the ballistic component and generation of the diffusive component. At very small noise intensities, one expects that the diffusion coefficient $D(\varepsilon)$ is increasing with

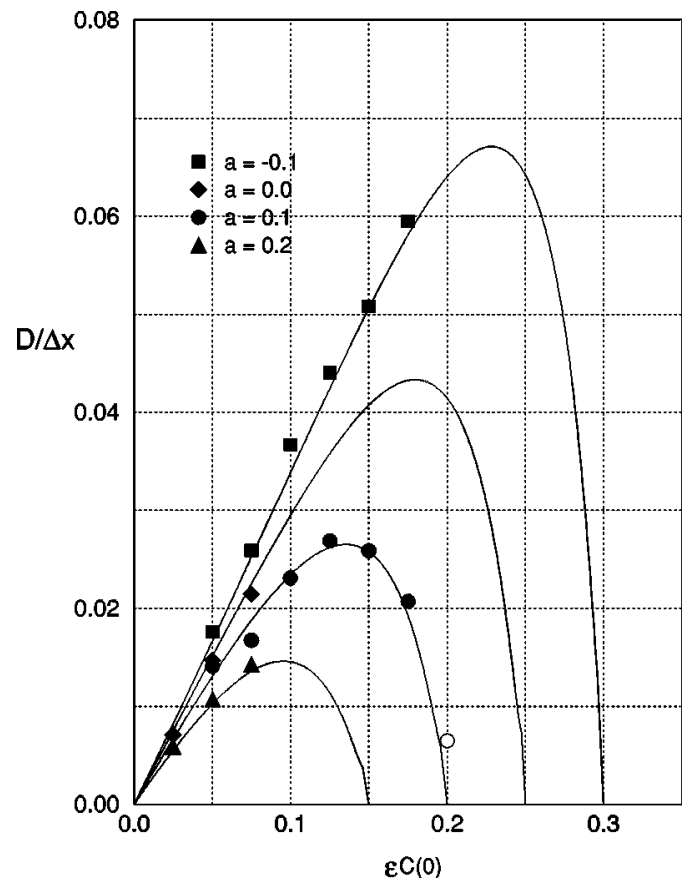

FIG. 2. Analytical and numerical results for the scaled diffusion coefficient, $D(\Delta x)^{-1}$, for different values of the external control parameter $a$ (see figure legend) and the effective noise intensity. Black symbols correspond to numerical simulations performed with $\Delta x=0.5$, and hollow symbols to those with $\Delta x=0.2$. In both cases we used $\Delta t=10^{-2}$. Results were obtained by averaging $10^{3}$ fronts evolving from the same initial steplike profile in the time window from $t_{i}=500$ to $t_{f}=1000$.

noise intensity. According to Eq. (21), however, it is clear that the mechanism by which the slow fluctuations of the field $\phi$ generate velocity fluctuations, which in turn produce the front diffusion, is sensitive to the actual mean front velocity. For larger front velocities, such fluctuations are more easily smoothed out. Since the front velocity itself is an increasing function of noise intensity, the combined result is that $D(\varepsilon)$ may decrease at sufficiently large noise intensities.

A brief comparison with a previous method is in order here. The small-noise-expansion approach [7,2,3] yields, at leading order in the noise intensity, a simple linear behavior which corresponds to the same Eq. (21) but with the deterministic front profile and velocity inside the integral. That result is thus recovered if the small noise expansion is performed directly on our result Eq. (21). The novelty of our result relies on the fact that contributions from all orders are included in the mean front profile and velocity, and a nontrivial interplay between the two effects of noise is thus obtained.

Note that the analytical result (21) yields $D(\varepsilon)=0$ in the linear regime $[1 / 2-2 \varepsilon(0) \leqslant a]$. This result points out that the assumption of diffusive spreading does not hold in the linear case, although it does not invalidate our previous results on the propagation velocity shift. Preliminary numerical simulations show that the stochastic spreading in this linear regime appears to be subdiffusive, i.e., $\left\langle\Delta z^{2}\right\rangle \sim t^{\alpha}$ with $\alpha$ $<1$. The characterization of this regime remains open.

\section{NOISE-SUSTAINED FRONT SOLUTIONS}

In this section we want to call attention to interesting features of front dynamics in the presence of multiplicative 


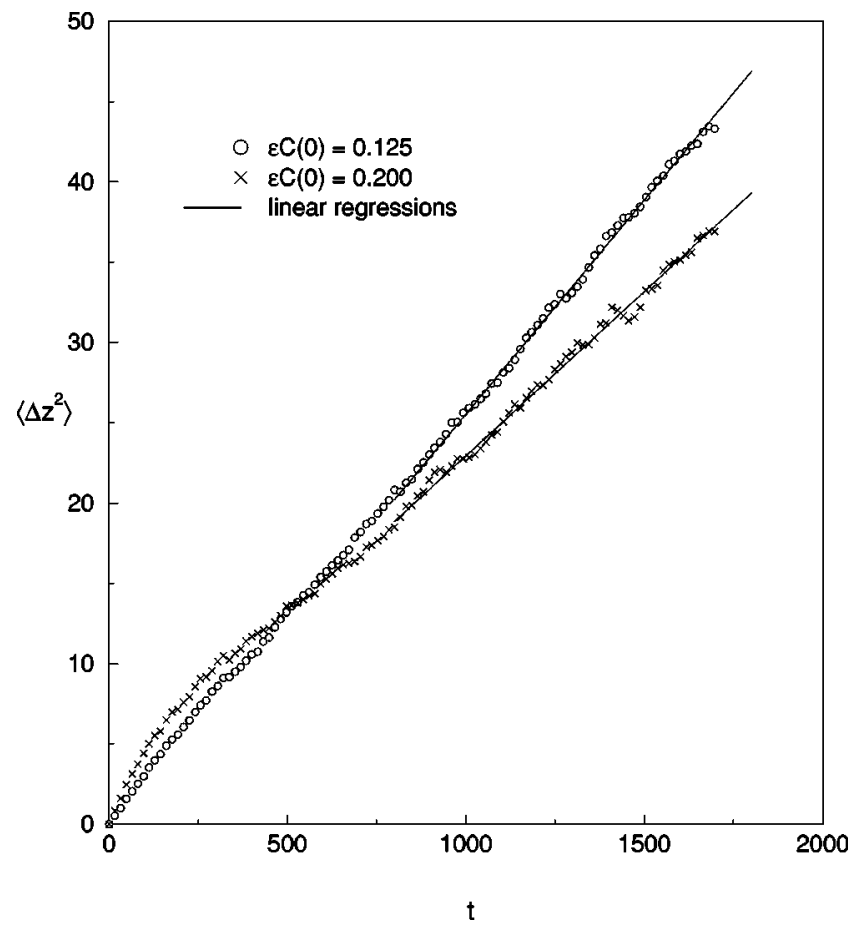

FIG. 3. Time evolution of the ensemble average $\left\langle\Delta^{2}(t)\right\rangle$ for $a$ $=0.1$ and two different values of the effective noise intensity. Data have been obtained by averaging $10^{3}$ fronts evolving from the same initial steplike profile with $\Delta x=0.5$ and $\Delta t=0.1$. Thin solid lines correspond to linear regressions of data evaluated long after the initial transient. The figure illustrates the bounded behavior of the diffusion spreading. For $\varepsilon C(0)=0.12$ (circles), $D$ present a larger slope than for $\varepsilon C(0)=0.20$ (diamonds).

noise which are associated with noise-induced transitions between the different regions in parameter space corresponding to the distinct regimes of front propagation. As discussed in Ref. [6], the location of the boundaries between the different regions is modified by noise, while the qualitative general picture is preserved. As a consequence, the introduction of noise in points of parameter space which are relatively close to such boundaries may change the qualitative behavior of the front dynamics.

The typical situation in all parameter space is that, if noise is introduced, the front velocity jumps to a larger value, and as soon as noise is switched off, the original velocity is recovered [6]. On the other hand, in the linear marginal stability region a continuum of possible velocities exists, which can be observed if the system is prepared with an initial condition with a (sufficiently slow) arbitrary decay of the leading edge. This latter situation occurs, for instance, if the system undergoes a sudden change of an external control parameter. However, if that quench is into the metastable region, the front solution will decay to the unique solution of that case.

The example we want to briefly discuss here is one in which noise can sustain solutions from the continuum of possible velocities in situations where the deterministic model has a unique solution. This will happen when the suitable control parameter has a value such that, in the absence of noise, the system is in the metastable region, while the presence of noise places it into the linear region. Suppose, for instance, that the front is driven to such a state in the

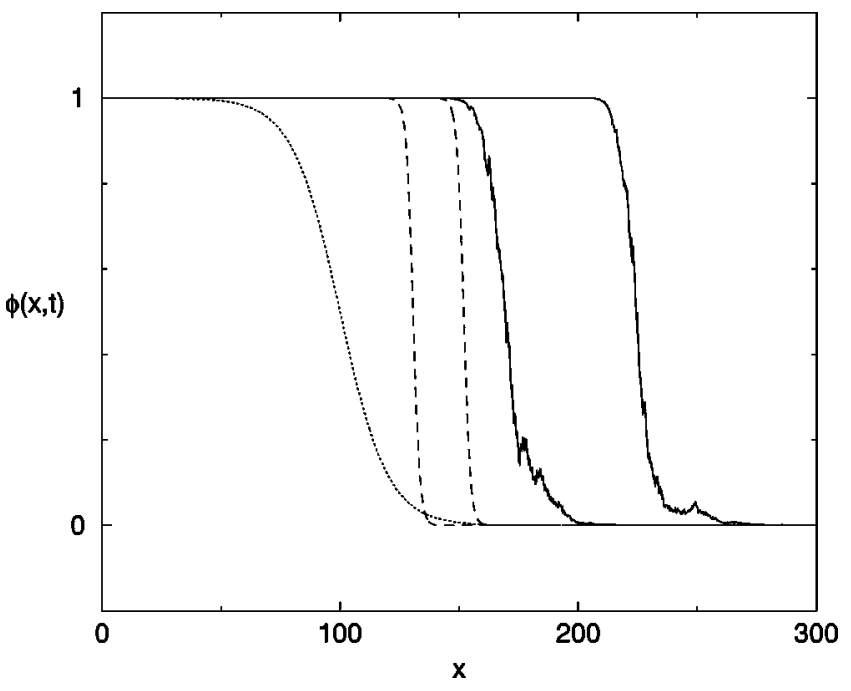

FIG. 4. Two stages in time, $t=50$ and $t=100$, of a deterministic front (dashed lines) and a noisy front (solid lines) from the same initial profile with $k_{i}=0.1$. Simulations were performed with $a$ $=-0.2, \varepsilon C(0)=0.3, \Delta x=0.1$, and $\Delta t=10^{-3}$.

presence of noise via a quench from the linear marginal stability region with a slow-decaying initial condition. In that case we will be able to sustain fronts with velocities within a continuous interval. Remarkably enough, this will be occurring in a situation in which, without noise, a unique velocity is allowed. Notice that the preparation procedure is crucial. By quenching the control parameter from the linear region, we take advantage of the fact that the solutions with larger velocities than the minimal one are also self-sustained.

This effect is shown in Fig. 4, where we plot profiles resulting from simulations of Eq. (23) with $a=-0.2$ for noise intensities $\varepsilon C(0)=0.3$ and $\varepsilon C(0)=0$. In both cases the initial condition is a slow-decaying profile with $k_{i}=0.1$. Whereas the deterministic case converges to the single nonlinear solution with a fast decay $k_{\mathrm{nl}}=1 / \sqrt{2}$, Eq. (28), and velocity $v_{\mathrm{nl}}=0.42$, Eq. (27), in the noisy case fluctuations sustain the initial slow decay $k_{i}$ and the velocity is $\bar{v}=1.10$ as given by Eq. (32).

\section{SUMMARY AND CONCLUSIONS}

We have constructed a nonperturbative scheme which is based on a multiple scale procedure to study front dynamics under external multiplicative fluctuations. The method allows us to formally separate the effects of noise on the ballistic and the diffusive components of the front propagation. On the ballistic component it predicts a change in the front velocity but it preserves the scenario of front selection for deterministic propagation, with shifts in the bondaries between different regions. The extension of the method to characterize the wandering of the front position relies on the assumption that this is diffusive. The self-consistency of the method predicts that the diffusive assumption is not always valid, but the method gives an explicit prediction for the diffusion coefficient when it exists.

The analytical predictions of our approach are not systematic but improve significantly the previous results based upon small noise expansions. Our results include a partial resum- 
mation of higher orders in the noise intensity, which turn out to capture the dominant physical behavior for a wide range of noise intensities, much beyond the validity of previous results. This has been tested numerically for a particular model, with very good agreement between theory and simulation.

The effect of multiplicative noise on the front velocity was already discussed in Ref. [6], of which the present paper is an extension. Our main specific result here is the explicit prediction of the diffusion coefficient of the front wandering. Its most salient feature is that it is not a monotonic increasing function of noise intensity. The mechanism by which slow fluctuations produce this wandering turns out to be affected by the actual mean front velocity, which in turn is affected by noise. Such a mechanism appears to be less effective for larger velocities. Since for moderately large noise intensities the front velocity increases significantly, the combined effect may result in an actual decrease of the front diffusion coefficient with increasing noise intensity.

It is also remarkable that the diffusion coefficient is reminiscent of the existence of a microscopic cutoff which regularizes the white noise. In the effective equation that is obtained for the ballistic component, the coefficients are renormalized with corrections which depend on the combination $\varepsilon / \Delta x$ which we identify with the effective noise in- tensity. On the contrary, the diffusion coefficient depends separately on $\varepsilon / \Delta x$ and $\varepsilon$. This reflects the fact that the models under consideration do not have a well defined continuum limit with white noise, and therefore results depend in general on the existence of a microscopic scale of noise. Such an additional length scale must in fact be an additional parameter of the problem. Furthermore, this clearly indicates that the correlation length of noise is an important parameter and may change drastically the scenario discussed here when it becomes comparable to or larger than the front width.

Finally, we have discussed the existence of noisesustained front solutions, in which the presence of noise makes accessible a continuum of solutions in situations in which this is not possible in the absence of noise.

\section{ACKNOWLEDGMENTS}

We acknowledge financial support from Dirección General de Investigación Científica y Técnica (Spain) (Projects PB96-0241 and PB96-1001-C02-02), and Commisionat per a Universitats i Recerca (Spain) (Project 1997SGR00439). We also acknowledge computing support from Fundació Catalana per a la Recerca-Centre de Supercomputació de Catalunya (Spain).
[1] Proceedings of the Workshop on External Noise and Its Interaction with Spatial Degrees of Freedom in Nonlinear Dissipative Systems, edited by C. R. Doering, H. R. Brand, and R. E.Ecke, J. Stat. Phys. 54, 1111 (1989).

[2] L. Schimansky-Geier and Ch. Zülicke, Z. Phys. B 82, 157 (1991).

[3] F. de Pasquale, J. Gorecki, and J. Popielawski, J. Phys. A 25, 433 (1992).

[4] J. Riordan, C. R. Doering, and D. Ben-Avraham, Phys. Rev. Lett. 75, 565 (1995).

[5] A. Lemarchand, A. Lesne, and M. Mareschal, Phys. Rev. E 51, 4457 (1995).

[6] J. Armero, J. M. Sancho, J. Casademunt, A. M. Lacasta, L.
Ramírez-Piscina, and F. Sagués, Phys. Rev. Lett. 76, 3045 (1996).

[7] A. S. Mikhailov, L. Schimansky-Geier, and W. Ebeling, Phys. Lett. 96A, 453 (1983).

[8] W. van Saarloos, Phys. Rev. Lett. 58, 2571 (1987); Phys. Rev. A 37, 211 (1988); 39, 6367 (1989).

[9] E. A. Novikov, Zh. Eksp. Teor. Fiz. 47, 1919 (1964) [Sov. Phys. JETP 20, 1290 (1965)].

[10] W. van Saarloos, M van Hecke, and R. Holyst, Phys. Rev. E 52, 1773 (1995).

[11] O. T. Valls and G. F. Mazenko, Phys. Rev. B 34, 7941 (1986).

[12] L. Ramírez-Piscina, J. M. Sancho, and A. HernándezMachado, Phys. Rev. B 48, 125 (1993). 